\title{
Domain walls in Born-Infeld-dilaton background
}

\author{
Debaprasad Maity* \\ The Institute of Mathematical Sciences, \\ C.I.T. Campus, Taramani, \\ Chennai - 600 113, India
}

December 8, 2018

\begin{abstract}
We study the dynamics of domain walls in Einstein-Born-Infeld-dilaton theory. Dilaton is non-trivially coupled with the Born-Infeld electromagnetic field. We find three different types of solutions consistent with the dynamic domain walls. For every case, the solutions have singularity. Further more, in these backgrounds, we study the dynamics of domain walls. We qualitatively plot various form of the bulk metrics and the potential encountered by the domain walls. In many cases, depending upon the value of the parameters, the domain walls show bouncing universe and also undergo inflationary phase followed by standard decelerated expansion.
\end{abstract}

PACS number: 04.50.-h, 04.60.Cf, 04.20.Jb

\section{Introduction}

Our universe as a four dimensional domain wall [1-9] in an extra dimensional spacetime is an interesting field to study particularly in the cosmological context. The domain wall is allowed to move in an extra spacelike dimension. The inherent nexus between this dynamics of the domain wall and Hubble like expansion equation of its scale factor attracts much attention to study the cosmology in a new perspective. In this scenario, all the standard model fields are assumed to be either in the bulk but dynamically peaked at the position of the wall [3, 6] or [10] fully localized on the domain wall depending upon what kind of model we are going to construct.

As we know for the past few years, domain wall are actively considered to embed as a four dimensional Friedmann-Robertson-Walker (FRW) universe in bulk spacetime with various possible matter components [11, 12]. Important aspect of this embedding, as just stated, is that boundary condition so called Israel junction condition[13] across the wall actually leads to Hubble expansion equation. So, what it turns out that various parameters of the bulk solutions effectively act as (invisible) energy density with different equations of states on the wall. So, by tuning these various bulk parameters in a model under consideration, one can in principle construct viable cosmology. In fact, on the

*E-mail: debu@imsc.res.in 
domain wall there exits bouncing cosmology. The important feature to mention in this kind of cosmology is non-singular transition between a contracting phase of the scale factor of the wall, and a following expanding stage [14, 15]. In this report will not going to construct the cosmological model. We will first try to find out the various possible background configurations in a string inspired theoretical model and then do a qualitative analysis on the dynamic domain walls in those backgrounds.

As is well known, Born-Infield type generalisation of abelian and non-abelian gauge field theories have long been the subject of interest in the context of superstring theory. For the abelian case, it was first argued in the reference [16] to be an open string effective action taking into account all order loop corrections in $\alpha^{\prime}$ (string coupling). On the other hand, it has also been noted that the D-branes [17] and some soliton solutions of supergravity, are governed by the Born-Infeld action. For various reasons particularly in the context of ADS/CFT correspondence, extending the gravitational background by including Born-Infeld gauge field in addition to Einstein-Hilbert term, has recently been considered extensively in [18, 19]. In a slightly different direction, we will try to analyse the dynamics of the domain wall in these background.

So, as a follow up of [20], here we will be discussing on dynamic domain walls in the more general Einstein-Born-Infield-dilaton [21] background along the line of [22]. String theories in their low-energy supergravity limit gives rise to effective models of gravity in higher dimensions which involve an infinite series of higher curvature terms in the gravity as well as gauge sector. For our purpose in this report, we will ignore the higher spacetime curvature terms. Although including the same is very much worth calculating. We leave this for future study (recent attempt towards this direction [23]). Considering this born-Infield Lagrangian, there has been considerable work on understanding the role of these higher derivative gauge field in various points of view, especially with regard to black hole physics [18, 19]. In this report we will analyze various static black hole as well as non-static spacetime solutions in consistent with dynamic domain walls in the aforementioned string inspired model. However, in addition to dilaton scalar field coupled with the BI Lagrangian in our theory, we also assume the dilaton to be coupled with domain wall in an exponential way [22]. We first analytically find three different types of metric solutions under specific relations among the various constant parameters in the theory. We have taken into account full back-reaction of the domain wall on background spacetime. Subsequently we study the structure of the solutions in details by plotting them in various possible region of the parameter space. In many cases, we find static black hole solution as well as various non-static solutions depending upon the choice of parameters. Then, we study the dynamics of a domain wall in those bulk backgrounds. A topic of particular interest in these kind of scenarios is how inflation occurs on the wall. In this regard, we find, for a wide range of parameter space of the model under consideration, the domain wall indeed inflates either in the early stage of the evolution followed by standard decelerated expansion or in asymptotic limit of its scale factor. Depending upon the choice of parameters, the inflation is either exponential or power law type with respect to the domain wall proper time. One important feature in these kind of scenarios is natural emergence of inflation as well as decelerated expansion phase on the domain wall world volume. Various energy densities which drive this dynamics, strictly come from the bulk.

We structured this report as follows: In the section 2, we start with an action corresponding to a domain wall moving in Born-Infield-dilaton background. We explicitly 
write down the equations of motion and corresponding boundary conditions across the domain wall. In section 3 , by taking static metric ansatz, we analytically solve the Einstein equations. We get three different types of solutions depending upon the value of the parameters in our theory. Due to complicated expressions, we study the structure of these solutions by graphical representation in various limits of radial coordinate. In section 4, again following the line of [22, 20], we plot the various potentials encountered by the domain walls and qualitatively study their dynamics. There are several situations we find, for which domain wall undergoes an inflationary phase as well as standard decelerated expansion. We also get bounce for finite value of scale factor as well as periodic universe on the domain wall world volume. This might lead to a hope of constructing the cosmological model in this theory also. Finally, in the section 5, we do some concluding remarks and describe some futures directions to work.

\section{Einstein equations and Boundary conditions}

We start with an string inspired action of Einstein-Born-Infeld system in an arbitrary spacetime dimension $n$. Along with this there exists a bulk scalar field $\phi$ called dilaton. The dilaton is assumed to have nontrivial coupling with the Born-Infeld field $A_{B}$ and a co-dimension one domain wall. The action takes the from

$$
S=\int d^{n} x \sqrt{-g}\left(\frac{1}{2} R-\frac{1}{2} \partial_{A} \phi \partial^{A} \phi-V(\phi)+\mathcal{L}(F, \phi)\right)+S_{D W},
$$

where action for the domain wall is

$$
S_{D W}=-\int d^{n-1} x \sqrt{-h}(\{K\}+\bar{V}(\phi)) .
$$

The expression for $\mathcal{L}(F, \phi)$ is

$$
L(F, \phi)=4 \lambda^{2} e^{2 \gamma \phi}\left(1-\sqrt{1+\frac{e^{-4 \gamma \phi} F^{A B} F_{A B}}{2 \lambda^{2}}}\right) .
$$

The constant $\lambda$ is the Born-Infeld parameter and has the dimension of mass. In the limit $\lambda \rightarrow \infty, L(F)$ reduces to the standard Maxwell form with a scalar field coupled exponentially like

$$
L(F)=-e^{-2 \gamma \phi} F^{A B} F_{A B}+\mathcal{O}\left(F^{4}\right) .
$$

$h_{A B}$ is the induced metric on the domain wall. $K$ is the trace of the extrinsic curvature of the domain wall. For simplicity, in this paper, we will work with the convention that $16 \pi G=1$, where $G$ is the Newton's constant.

By varying the action with respect to the gauge field $A_{B}$, dilaton field $\phi$ and the metric $g_{A B}$, we get the equations of motion as

$$
\begin{aligned}
& R_{A B}=\partial_{A} \phi \partial^{A} \phi+\frac{2}{n-2} V(\phi) g_{A B}+\frac{8 \lambda^{2}}{(n-2)} e^{2 \gamma \phi}\left\{2 \mathcal{Y} \frac{\partial \mathcal{L}}{\partial \mathcal{Y}}-\mathcal{Y}\right\} g_{A B}-8 e^{-2 \gamma \phi} \frac{\partial \mathcal{L}}{\partial \mathcal{Y}} F_{A C} F_{B}^{C}(4) \\
& D_{C} \partial^{C} \phi-\frac{\partial(\phi)}{\partial \phi}+8 \lambda^{2} \gamma e^{2 \gamma \phi}\left\{2 \mathcal{Y} \frac{\partial \mathcal{L}}{\partial \mathcal{Y}}-\mathcal{Y}\right\}=0 \\
& D_{A}\left(e^{-2 \gamma \phi} \frac{\partial \mathcal{L}}{\partial \mathcal{Y}} F^{A B}\right)=0
\end{aligned}
$$


where, $D_{A}$ is co-variant derivative with respect to the bulk metric and the new variable $\mathcal{Y}=\frac{e^{-4 \gamma \phi} F^{A B} F_{A B}}{2 \lambda^{2}}$. We also have the corresponding boundary conditions as follows

$$
\begin{aligned}
& \left\{K_{M N}\right\}=-\frac{1}{n-2} \bar{V}(\phi) h_{M N} \\
& \left\{n^{M} \partial_{M} \phi\right\}=\frac{\partial \bar{V}(\phi)}{\partial \phi}
\end{aligned}
$$

where, $n^{M}$ is the unit normal to the domain wall. $R$ is the curvature scalar. The first boundary condition comes from the Israel junction condition (for details [22]) across the wall.

We will be considering the solution which has reflection $\operatorname{symmetry}\left(Z_{2}\right)$ across the domain wall. So, from Eq.7, expression for the extrinsic curvature turns out to be

$$
K_{M N}=-\frac{1}{2(n-2)} \bar{V}(\phi) h_{M N}
$$

We consider the static spherically symmetric bulk metric ansatz as

$$
d s^{2}=-N(r) d t^{2}+\frac{1}{N(r)} d r^{2}+R(r)^{2} d \Omega_{\kappa}^{2}
$$

where, we have taken $d \Omega_{\kappa}^{2}$ as the line element on a $(n-2)$ dimensional space of constant curvature with the metric $\tilde{g}_{i j}$. The Ricci curvature of this sub-space is $\tilde{R}_{i j}=k(n-3) \tilde{g}_{i j}$ with $k \in\{-1,0,1\}$

We want to get the spherically symmetric solutions corresponding to a homogeneous and isotropic induced metric on the domain wall like

$$
d s_{w a l l}^{2}=-d \tau^{2}+R(\tau)^{2} d \Omega_{\kappa}^{2}
$$

which is Robertson-Walker metric. $\tau$ is the domain wall proper time. The size of the $n$-dimensional domain wall universe is determined by the radial distance, $R$, which in turn determines the position of it in the bulk spacetime.

However, by using the unit normal pointing into $r<r(t)$ and the unit tangent to the moving wall

$$
\begin{aligned}
n_{M} & =\frac{\sqrt{N}}{\sqrt{N^{2}-\dot{r}^{2}}}(\dot{r},-1,0, \ldots, 0), \\
u^{M} & =\frac{\sqrt{N}}{\sqrt{N^{2}-\dot{r}^{2}}}(1, \dot{r}, 0, \ldots, 0),
\end{aligned}
$$

respectively, one can express the induced metric on the domain wall and its extrinsic curvature as

$$
\begin{aligned}
& h_{M N}=g_{M N}-n_{M} n_{N} \\
& K_{M N}=h_{M}^{P} h_{N}^{Q} \nabla_{P} n_{Q} .
\end{aligned}
$$

Where $\dot{r}=\frac{\partial r}{\partial t}$. 
So, the expressions for the components of the extrinsic curvature come out to be

$$
\begin{aligned}
& K_{i j}=-\frac{R^{\prime}}{R} \frac{N^{3 / 2}}{\sqrt{N^{2}-\dot{r}^{2}}} h_{i j} \\
& K_{00}=\frac{1}{\dot{r}} \frac{d}{d t}\left(\frac{N^{3 / 2}}{\sqrt{N^{2}-\dot{r}^{2}}}\right) .
\end{aligned}
$$

'Prime' is derivative with respect to bulk radial coordinate $r$.

Using the equations for $K_{i j}$ into $K_{00}$, and then integrating one gets

$$
R^{\prime}=C \bar{V}(\phi) .
$$

Now, by using Eq17 in the boundary condition for the scalar field one gets

$$
\frac{\partial \phi}{\partial R}=-\frac{n-2}{R} \frac{1}{\bar{V}} \frac{\partial \bar{V}}{\partial \phi}
$$

This equation has to hold at every point in the bulk visited by the domain wall. So, if the wall visits a range of $\mathrm{R}$, then the above equation can be solved to yield $\phi$ as a function of $\mathrm{R}$ without specifying the bulk potential. This gives us a consistency condition for the dynamic domain wall coupled with the bulk scalar to exists. In what follows, we will use this condition to get final solution for the scalar field and the metric.

\section{The solutions for bulk metric}

In this section, we first solve the equation for Born-Infeld field. Then using this solution to the remaining equations of motion we will find out the solution for metric under static bulk metric ansatz Eq.10.

So, we note that a class of solutions for the equation of Born-Infield electromagnetic field can be written down with all the components of $F^{A B}$ being zero except $F^{r t}$. The solution looks like

$$
F^{r t}=\frac{2 Q \lambda e^{2 \gamma \phi}}{\sqrt{4 Q^{2}+\lambda^{2} R^{2 n-4}}}
$$

where, $Q$ is the integration constant and related to the electromagnetic charge. One can define electromagnetic charge with respect to an asymptotic observer as

$$
q=\frac{1}{4 \pi} \int_{\Sigma_{\infty}} e^{-2 \gamma \phi *} F=\frac{Q \omega_{n-1}}{4 \pi},
$$

where, ${ }^{*} F_{A B}=\frac{1}{2 \sqrt{-g}} \varepsilon^{A B C D} F^{C D}$ and $\Sigma_{\infty}$ is a hyper-surface at $R \rightarrow \infty . \omega_{n-1}$ is volume of unity $n$ sphere. One can notice that the electric field is finite at $R=0$. This is expected in Born-Infeld theories. 
Now using Eq,19] and the metric ansatz Eq,10, one can read out the remaining equations of motion as

$$
\begin{aligned}
& \frac{R^{\prime \prime}}{R}=-\frac{1}{n-2} \phi^{\prime 2} \\
& \frac{1}{2 R^{n-2}}\left\{N\left(R^{n-2}\right)^{\prime}\right\}^{\prime}-\frac{k(n-3)(n-2)}{2 R^{2}}=-V-\mathcal{T}_{22}(R, Q) \\
& \frac{n-2}{4 R^{n-2}}\left(N^{\prime} R^{n-2}\right)^{\prime}=-V-\mathcal{T}_{00}(R, Q) \\
& \frac{1}{R^{n-2}}\left(\phi^{\prime} N R^{n-2}\right)^{\prime}=\frac{\partial V(\phi)}{\partial \phi}+8 \lambda^{2} \gamma e^{2 \gamma \phi} \mathcal{F}(r, Q),
\end{aligned}
$$

where, various new notations are given below,

$$
\mathcal{T}_{22}(R, Q)=4 \lambda^{2} e^{2 \gamma \phi} \mathcal{F}(R, Q) \quad ; \quad \mathcal{T}_{00}(R, Q)=4(n-2) \lambda^{2} e^{2 \gamma \phi}\left[\frac{\mathcal{F}(R, Q)}{n-2}+\frac{\mathcal{G}(R, Q)}{2}\right]
$$

and

$$
\mathcal{F}(R, Q)=\frac{\sqrt{4 Q^{2}+\lambda^{2} R^{2 n-4}}}{\lambda R^{n-2}}-1 \quad ; \quad \mathcal{G}(R, Q)=-\frac{4 Q^{2}}{\sqrt{4 Q^{2}+\lambda^{2} R^{2 n-4}}} \frac{1}{\lambda R^{n-2}} .
$$

$\mathcal{T}_{00}$ and $\mathcal{T}_{22}$ are proportional to the $t t$ and spatial components of the energy-momentum tensor for the Born-Infield Lagrangian respectively.

Now, in order to find the solutions of Einstein equation, we will employ the Eqs.(1718). So, taking the Liouville type brane potential

$$
\bar{V}(\phi)=\bar{V}_{0} e^{\alpha \phi}
$$

one can easily get the solution for the scalar field without specifying the bulk potential, as well as for the radius $R(r)$ of the unit sphere $\Omega_{k}$ as

$$
\begin{aligned}
& \phi=\phi_{0}-\frac{\alpha(n-2)}{\alpha^{2}(n-2)+1} \log (r) \\
& R(r)=C \bar{V}_{0} e^{\alpha \phi_{0}} r^{\frac{1}{\alpha^{2}(n-2)+1}}
\end{aligned}
$$

where $\phi_{0}$ and $C$ are integration constants. Furthermore, in order to have the solution for the bulk metric, we need to specify the bulk potential. So, again we take the same Liouville type bulk potential,

$$
V(\phi)=V_{0} e^{\beta \phi}
$$

where, $V_{0}$ is constant. Now, by considering above two expressions Eqs.(25) for $R$ and $\phi$ as solutions ansatz and making use of the bulk potential for the scalar field, we find three different types of solutions corresponding to the full Einstein equations of motion. In what follows, we discuss about the nature of these various types of solutions and subsequently the dynamics of the domain walls in those bulk backgrounds.

Type-I solution: When, $\alpha=\beta=\gamma=0$. We note that the bulk and brane potential play the roll of cosmological constant and brane tension respectively. So, effectively, the action is a Einstein-Born-Infeld system with a bulk cosmological constant and domain wall with fixed tension. 
By choosing these particular set of value of the parameters, the solution turns out to be

$$
\begin{aligned}
N(r)= & k-2 M r^{-(n-3)}-\left(\frac{2 V_{0}}{(n-2)(n-1)}-\frac{8 \lambda^{2}}{(n-2)(n-1)}\right) r^{2} \\
+ & \frac{8 \lambda r^{-(n-4)}}{(n-1)(n-2)}\left(-\sqrt{4 Q^{2}+\lambda^{2} r^{2 n-4}}+\frac{4(n-2) Q^{2} r^{-(n-2)}}{\lambda(n-3)} \mathcal{D}(r, Q)\right) \\
& R(r)=r \quad ; \quad \phi=\phi_{0},
\end{aligned}
$$

Where $M$ and $\phi_{0}$ are integration constants and

$$
\mathcal{D}(r, Q)={ }_{2} F_{1}\left[\frac{n-3}{2 n-4}, \frac{1}{2}, \frac{3 n-7}{2 n-4},-\frac{4 Q^{2} r^{-(2 n-4)}}{\lambda^{2}}\right] .
$$

Now, as a whole expression, the solution looks much difficult. So, it is rather easer to see the metric in various limits of the radial coordinate and study its behaviour.

As we note, for large $r$ the expression for the above solution looks like

$\left.N(r)\right|_{r \rightarrow \infty}=k-\frac{2 V_{0}}{(n-2)(n-1)} r^{2}-2 M r^{-(n-3)}+\frac{16 Q^{2}}{(n-3)(n-2)} r^{-(2 n-6)}+\mathcal{O}\left(r^{\frac{10-4 n}{1+c^{2}}}\right),(31$

and for small $r$ limit,

$$
\begin{aligned}
\left.N(r)\right|_{r \rightarrow 0}=k-2 \mathcal{M}_{1} r^{-(n-3)} & -\frac{16 \lambda \mathcal{Q}_{1}}{(n-1)(n-2)} r^{-(n-4)}-\frac{2 \mathcal{V}_{1}}{(n-1)(n-2)} r^{2} \\
& -\frac{8 \lambda \mathcal{H}_{1}}{(n-1)(n-2)} r^{n}+\mathcal{O}\left(r^{3 n-4}\right),
\end{aligned}
$$

where

$$
\begin{aligned}
\mathcal{M}_{1} & =M-\frac{16 Q^{2} \Gamma\left[\frac{3 n-7}{2 n-4}\right] \Gamma\left[\frac{1}{2 n-4}\right]}{\sqrt{\pi}(n-1)(n-3)}\left(\frac{4 Q^{2}}{\lambda^{2}}\right)^{-\frac{n-3}{2 n-4}} \\
\mathcal{Q}_{1} & =Q-\frac{2 Q^{2}(n-2) \Gamma\left[\frac{3 n-7}{2 n-4}\right] \Gamma\left[\frac{-1}{2 n-4}\right]}{\lambda(n-3) \Gamma\left[\frac{n-3}{2 n-4}\right] \Gamma\left[\frac{2 n-5}{2 n-4}\right]}\left(\frac{4 Q^{2}}{\lambda^{2}}\right)^{-\frac{1}{2}} \\
\mathcal{H}_{1} & =\frac{\lambda^{2}}{4 Q}+\frac{1}{(2 n-3)}\left(\frac{4 Q^{2}}{\lambda^{2}}\right)^{-1}\left(Q-\mathcal{Q}_{1}\right) \\
\mathcal{V}_{1} & ==V_{0}-4 \lambda^{2} .
\end{aligned}
$$

The solution for the scalar field becomes constant [18]. In order to have better understanding of the metric, we have plotted the metric in the Fig.1 for several possibilities of parameter values. Now it is easy to read off the horizon structure from the figure. As is seen from the figures that for every case, there exists singularity at $r=0$ which is either timelike or spacelike.

We have four possibilities for different region of the parameter space $\left(V_{0}, M\right)$. When $V_{0}>0, M>0$, for the first case the metric would be either a Reisner-Nordstrom(RN) black hole inside the cosmological horizon $(k=1)$ or only de Sitter space $(k=0,-1)$. For the other case, we have either Schwarzschild-de Sitter black hole which has black hole $(k=1)$ and cosmological horizon or non-static spacetime $(k=0,-1)$ with a naked 

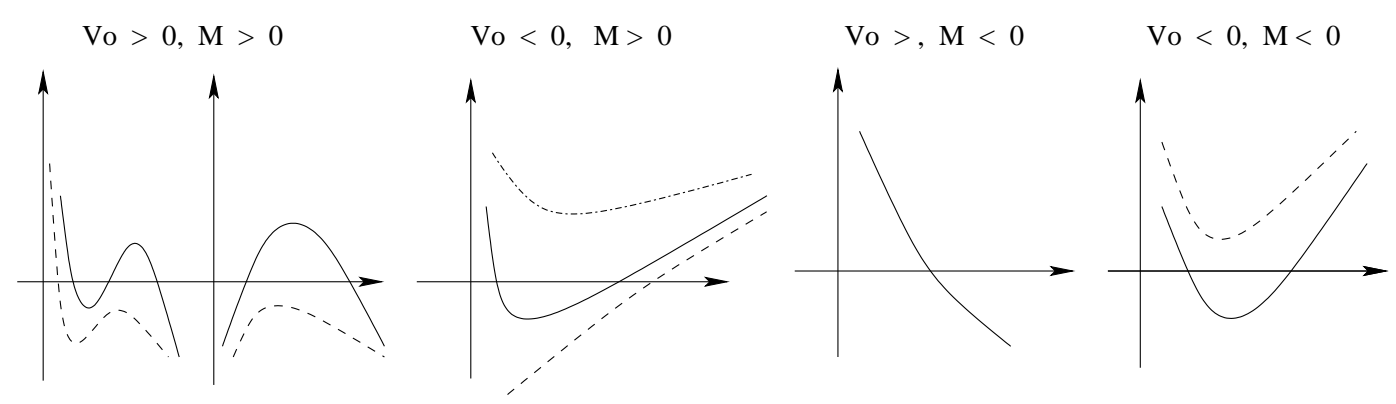

Figure 1: N(r) for type-I solution.

singularity. As is clear, for every case, the behaviour of the metric near the singularity region $r \rightarrow 0$ is governed by the sign of $\mathcal{M}_{1}$. In all these cases asymptotically, the metric becomes de-sitter space where, $V_{0}$ is playing the roll of cosmological constant. Usually, the ADM [24] mass of the black hole is taken to be

$$
m_{A D M}=\frac{2 n \omega_{n-1}}{2} M
$$

where, $\omega_{n-1}$ is the volume of the unit $n$-sphere.

When $V_{0}>0, M<0$, the singularity is either space-like or time like defined by the sign of $\mathcal{M}_{1}$, negative or positive respectively. The interplay between the value of $Q$ and $M$ determines the singularity structure at $r=0$. Asymptotically the metric is Anti-de Sitter. For one case we have Reisner-Nordstrom (RN) black hole or there is a naked singularity at $r=0$. For the other case, the spacetime is Schwarzschild-anti-de Sitter.

If $V_{0}<0, M>0$, for every case $k=0, \pm 1$, the metric has cosmological horizon with asymptotically de Sitter space.

When $V_{0}<0, M<0$, for $k=0,1$, the metric has a time like naked singularity at $r=0$ for any value of the other parameters present. However, $k=1$ leads to a possibility of having a RN black hole in an asymptotically Anti-de Sitter spacetime for certain range of parameter space $\left(V_{0}, M\right)$ and $\lambda Q^{2}$.

Type-II solution: For $\alpha=\frac{\beta}{2}=\gamma ; k=0$, which means that metric with flat spatial section is the only allowed configuration for this choice of parameters. The solution comes out to be

$$
\begin{aligned}
N(r)= & -\left(1+c^{2}\right)^{2} r^{\frac{2}{1+c^{2}}}\left[\frac{1}{\left(n-1-c^{2}\right)}\left\{2 L_{0}-\frac{8 \lambda^{2} \Omega}{(n-2)\left(1+c^{2}\right)^{2}}\right\}+2 M r^{-\frac{n-1-c^{2}}{1+c^{2}}}\right] \\
& +\frac{8 \lambda \Omega r^{-\frac{n-4}{1+c^{2}}}}{(n-2)\left(1+c^{2}-n\right)}\left(\sqrt{4 Q^{2}+\lambda^{2} r^{\frac{2 n-4}{1+c^{2}}}}-\frac{4(n-2) Q^{2} r^{-\frac{n-2}{1+c^{2}}}}{\lambda\left(-3+c^{2}+n\right)} \mathcal{P}(r, Q)\right) \\
& R(r)=r^{\frac{1}{1+c^{2}}} ; \quad ;(r)=\sqrt{n-2}\left(\phi_{0}^{*}-\frac{b}{1+c^{2}} \log (r)\right)
\end{aligned}
$$

where $\phi_{0}^{*}=\phi_{0} / \sqrt{n-2}$, the integration constants. Various other notations are given below,

and

$$
c=\frac{1}{2} \beta \sqrt{n-2} \quad ; \quad L_{0}=\frac{V_{0} e^{2 c \phi_{0}^{*}}}{n-2} \quad ; \quad \Omega=\frac{\left(1+c^{2}\right)^{2} e^{2 c \phi_{0}^{*}}}{n-2}
$$

$$
\mathcal{P}(r, Q)={ }_{2} F_{1}\left[\frac{c^{2}+n-3}{2 n-4}, \frac{1}{2}, \frac{3 n-7+c^{2}}{2 n-4},-\frac{4 Q^{2} r^{-\frac{2 n-4}{1+c^{2}}}}{\lambda^{2}}\right]
$$




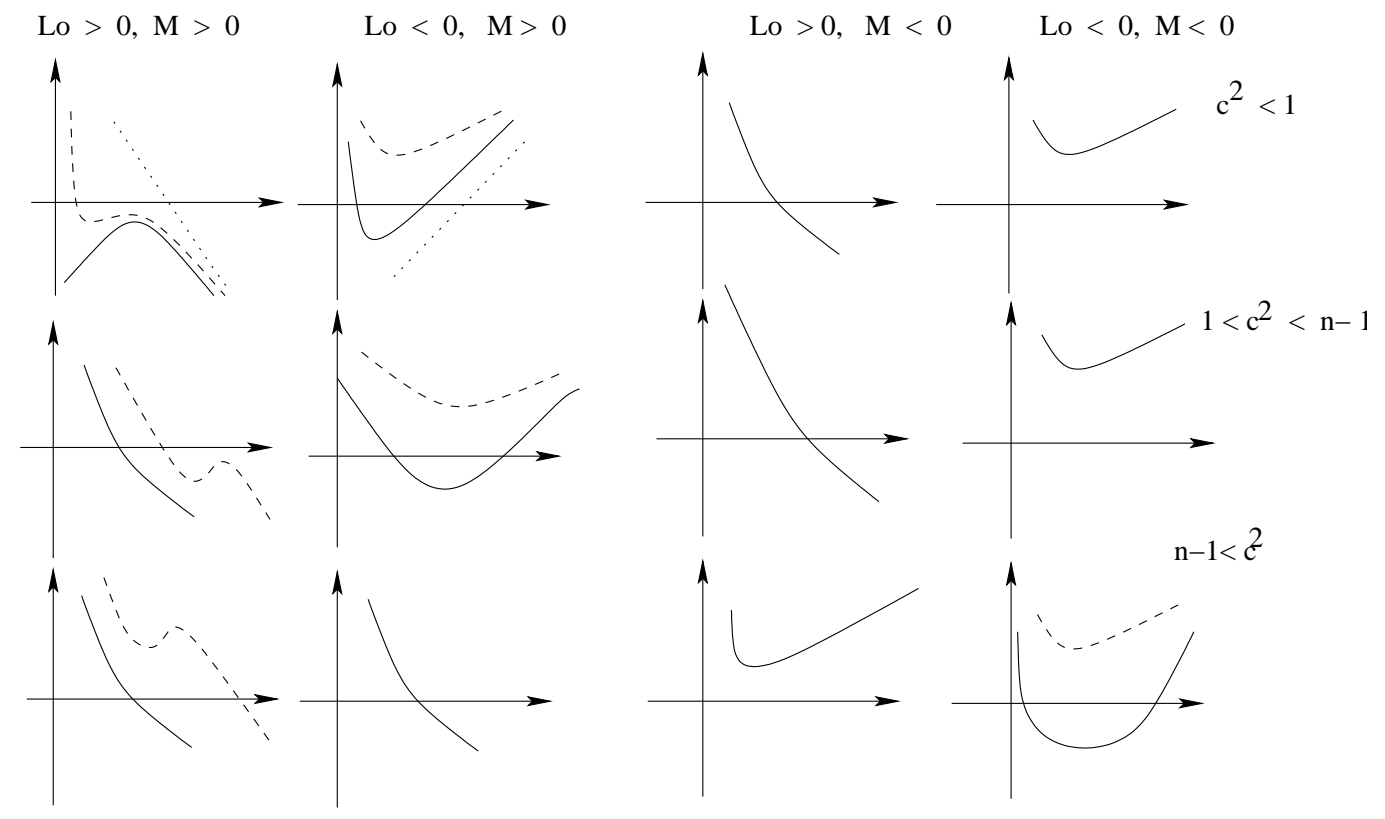

Figure 2: N(r) for type-II solution.

Again, taking the large $r$ limit, the above solution turns out to be

$$
\frac{\left.N(r)\right|_{r \rightarrow \infty}}{\left(1+c^{2}\right)^{2}}=r^{\frac{2}{1+c^{2}}}\left[\frac{2 L_{0}}{\left(1+c^{2}-n\right)}-2 M r^{-\frac{n-1-c^{2}}{1+c^{2}}}\right]+\frac{16 \Omega^{\prime} Q^{2}}{\left(n-3+c^{2}\right)} r^{-\frac{2 n-6}{1+c^{2}}}+\mathcal{O}\left(r^{\frac{10-4 n}{1+c^{2}}}\right)
$$

and for small $r$ limit,

$$
\begin{aligned}
\frac{\left.N(r)\right|_{r \rightarrow 0}}{\left(1+c^{2}\right)^{2}}=-2 \mathcal{M}_{2} r^{-\frac{n-3-c^{2}}{1+c^{2}}} & -\frac{16 \lambda \Omega^{\prime} \mathcal{Q}_{2}}{\left(n-1-c^{2}\right)} r^{-\frac{n-4}{1+c^{2}}}-\frac{2 \mathcal{V}_{2}}{n-1-c^{2}} r^{\frac{2}{1+c^{2}}} \\
& -\frac{8 \lambda \Omega^{\prime} \mathcal{H}_{2}}{\left(n-1-c^{2}\right)} r^{\frac{n}{1+c^{2}}}+\mathcal{O}\left(r^{\frac{3 n-4}{1+c^{2}}}\right),
\end{aligned}
$$

where

$$
\begin{aligned}
\mathcal{M}_{2} & =M-\frac{16 Q^{2} \Omega^{\prime}(n-2) \Gamma\left[\frac{3 n-7+c^{2}}{2 n-4}\right] \Gamma\left[\frac{1-c^{2}}{2 n-4}\right]}{\sqrt{\pi}\left(n-1-c^{2}\right)\left(n-3+c^{2}\right)}\left(\frac{4 Q^{2}}{\lambda^{2}}\right)^{-\frac{n-3+c^{2}}{2 n-4}} \\
\mathcal{Q}_{2} & =Q-\frac{Q(n-2) \Gamma\left[\frac{3 n-7+c^{2}}{2 n-4}\right] \Gamma\left[\frac{c^{2}-1}{2 n-4}\right]}{\left(n-3+c^{2}\right) \Gamma\left[\frac{n-3+c^{2}}{2 n-4}\right] \Gamma\left[\frac{2 n-5+c^{2}}{2 n-4}\right]} \\
\mathcal{H}_{2} & =\frac{\lambda^{2}}{4 Q}+\frac{\left(1-c^{2}\right)}{\left(2 n-3-c^{2}\right)}\left(\frac{4 Q^{2}}{\lambda^{2}}\right)^{-1}\left(Q-\mathcal{Q}_{2}\right) \\
\mathcal{V}_{2} & ==L_{0}-4 \lambda^{2} \Omega^{\prime} \\
\Omega^{\prime} & =\frac{e^{2 c \phi_{0}^{*}}}{n-2} .
\end{aligned}
$$

For this case again, we have plotted various possibilities for different values of the parameters present in the expression for $N(r)$. All the detailed structure can easily be read off from the corresponding Fig,2. 
The structure of the space time is depending upon the value of $c$. If $c^{2}<1$, the singularity behaviour is determined by the $\operatorname{sign}$ of $\mathcal{M}_{2}$. As is seen from the expression of $\mathcal{M}_{1}$, for $M<0$, it is always negative which leads to timelike singularity at $r=0$. Whereas for $M>0$, the metric can have both types of singularities depending upon the value of other parameters $Q$ and $\lambda$. This leads us to a quite distinct singularity behaviour which is expected in a Born-Infeld theory as opposed to the standard RN black hole spacetime in Einstein-Maxwell theory. In particular, the spacetime singularity comes from a mass dependent term in the metric.

On the other hand, for $c^{2}>1, \mathcal{Q}_{2}$ determines the singularity behaviour. It is interesting to note that the expression for $\mathcal{Q}_{2}$ is such, the combined expression $\frac{\mathcal{Q}_{2}}{\left(n-1-c^{2}\right)}$ is always negative. This in turn leads to timelike singularity for any value of $c^{2}>1$ as is also clear from the plots Fig, 2 .

However, for a wide range of parameter space, the metric has a cosmological horizon. For $c^{2}<1$, and $1<c^{2}<n-1$, asymptotic structure of the spacetime is depending upon the value of $L_{0}$ which is playing the roll of cosmological constant. On the other hand for $c^{2}>n-1$, it is governed by the sign of the parameter $M$. As we note the asymptotic expression for the metric to be

$$
\begin{array}{ll}
d s^{2}=r^{\frac{2}{1+c^{2}}}\left(-L_{0} d t^{2}+d \Omega_{k}^{2}\right)+\frac{1}{L_{0} r^{\frac{2}{1+c^{2}}}} d r^{2} \quad \text { for } c^{2}<n-1 \\
d s^{2}=r^{\frac{2}{1+c^{2}}}\left(-h(r) d t^{2}+d \Omega_{k}^{2}\right)+\frac{1}{h(r) r^{\frac{2}{1+c^{2}}}} d r^{2} & \text { for } c^{2}>n-1,
\end{array}
$$

where

$$
h(r)=\left(\frac{2 L_{0}}{n-1-c^{2}}+2 M r^{-\frac{n-1-c^{2}}{1+c^{2}}}\right) .
$$

So, for the second case, we observe that the spacetime is asymptotically ADS kind of Schwarzschild black hole for the parameter $L_{0}>1, M<0$ as also depicted in the plot. Now, for both the parameters $M, L_{0}$ being negative, the bulk metric would be globally non-static with a naked singularity. On the other hand when $L_{0}<0, M>0$, the second metric Eq $\$ 51$ has a cosmological horizon.

Now, for $c^{2}<1$ and $L_{0}<0, M>0$, the metric can have either a RN or Schwarzschild black hole depending upon the value of $\mathcal{M}_{2}$. Whereas for $1>c^{2}>n-1$ we can have again a RN black hole in the bulk. But for both the cases, the bulk metric can have naked singularity depending upon the region of the parameter space.

Type-III solution: For $\alpha=\frac{2}{\beta(n-2)}=\gamma ; k \neq 0$, the metric has no solution with flat spatial section. The solution looks like

$$
\begin{aligned}
N(r)= & \left(1+c^{2}\right)^{2} r^{\frac{2}{1+c^{2}}}\left[\frac{-2 L_{0}}{(n-3) c^{2}+1}-2 M r^{-\frac{(n-3) c^{2}+1}{1+c^{2}}}\right]+\frac{8 \lambda^{2} \Omega r^{\frac{2 c^{2}}{1+c^{2}}}}{c^{2}\left((n-1) c^{2}-1\right)} \\
- & \frac{8 \lambda \Omega r^{-\frac{(n-4) c^{2}}{1+c^{2}}}}{c^{2} \xi^{n-2}\left((n-1) c^{2}-1\right)}\left(\sqrt{4 Q^{2}+\lambda^{2} R^{2 n-4}}-\frac{4(n-2) c^{2} Q^{2} r^{-\frac{(n-4) c^{2}}{1+c^{2}}}}{\lambda \xi^{n-2}\left((n-3) c^{2}+1\right)} \Delta(r, Q)\right) \\
& R(r)=\xi r^{\frac{c^{2}}{1+c^{2}}} ; \phi(r)=\sqrt{n-2}\left(\phi_{0}^{*}-\frac{c}{1+c^{2}} \log (r)\right)
\end{aligned}
$$




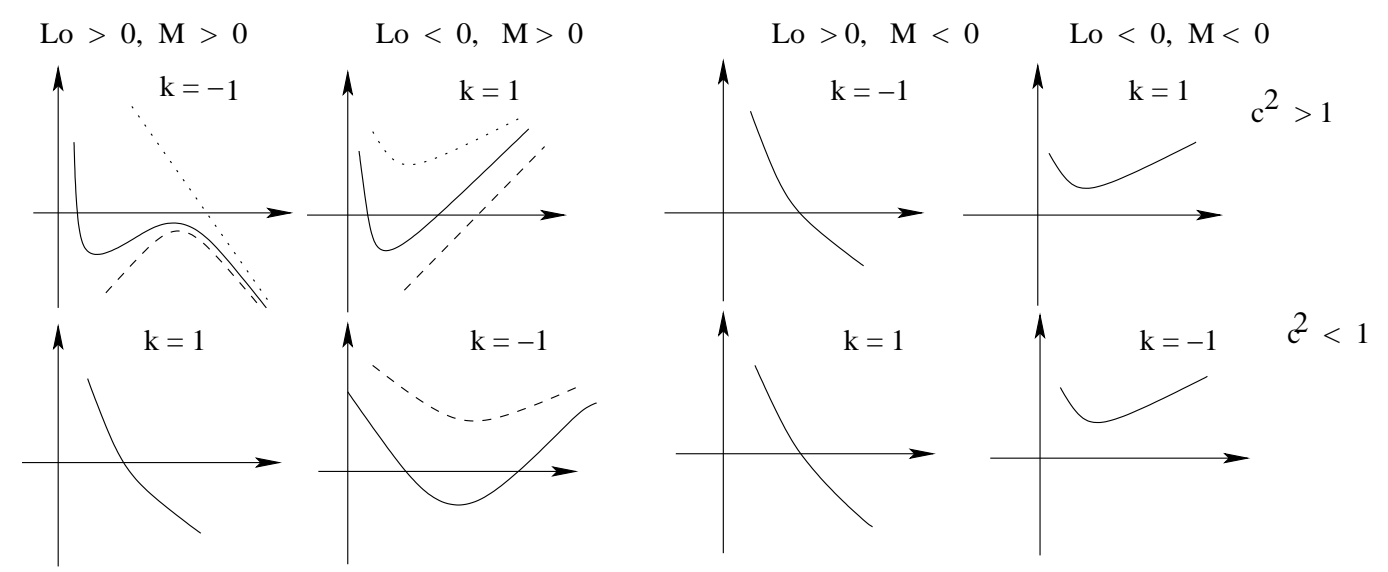

Figure 3: N(r) for type-III solution

where, we use the notation

$\xi=\sqrt{\frac{k(n-3)}{2 L_{0}\left(1-c^{2}\right)}} \quad ; \quad \Delta(r, Q)={ }_{2} F_{1}\left[\frac{(n-3) c^{2}+1}{c^{2}(2 n-4)}, \frac{1}{2}, \frac{(3 n-7) c^{2}+1}{(2 n-4) c^{2}},-\frac{4 Q^{2} R^{(4-2 n)}}{\lambda^{2}}\right]$

For large $r$ limit, expression for the above solution terns out to be

$\frac{\left.N(r)\right|_{r \rightarrow \infty}}{\left(1+c^{2}\right)^{2}}=\frac{-2 L_{0} r^{\frac{2}{1+c^{2}}}}{\left((n-3) c^{2}+1\right)}-2 M r^{-\frac{\left((n-3) c^{2}-1\right)}{1+c^{2}}}+\frac{16 \Omega^{\prime} Q^{2} r^{-\frac{(2 n-6) c^{2}}{1+c^{2}}}}{c^{2} \xi^{2 n-4}\left((n-3) c^{2}+1\right)}+\mathcal{O}\left(r^{\frac{(10-4 n) c^{2}}{1+c^{2}}}\right)$

whereas in small $r$ limit, it will be

$$
\begin{aligned}
\frac{\left.N(r)\right|_{r \rightarrow 0}}{\left(1+c^{2}\right)^{2}}= & -2 \mathcal{M}_{3} r^{-\frac{(n-3) c^{2}-1}{1+c^{2}}}-\frac{16 \lambda \Omega^{\prime} \mathcal{Q}_{3}}{c^{2} \xi^{n-2}\left((n-1) c^{2}-1\right)} r^{-\frac{(n-4) c^{2}}{1+c^{2}}}-\frac{2 V}{(n-1) c^{2}+1} r^{\frac{2}{1+c^{2}}} \\
& +\frac{8 \beta^{2} \Omega^{\prime} r^{\frac{2 c^{2}}{1+c^{2}}}}{c^{2}\left((n-1) c^{2}-1\right)}-\frac{8 \lambda \Omega^{\prime} \mathcal{H}_{3} r^{\frac{n c^{2}}{1+c^{2}}}}{c^{2}\left((n-1) c^{2}-1\right)}+\mathcal{O}\left(r^{\frac{(3 n-4) c^{2}}{1+c^{2}}}\right)
\end{aligned}
$$

Various notations are in order

$$
\begin{aligned}
\mathcal{M}_{3} & =M-\frac{16 Q^{2} \Omega^{\prime}(n-2) \Gamma\left[\frac{(3 n-7) c^{2}+1}{(2 n-4) c^{2}}\right] \Gamma\left[\frac{c^{2}-1}{(2 n-4) c^{2}}\right]}{\sqrt{\pi} \xi^{2 n-4}\left((n-1) c^{2}-1\right)\left((n-3) c^{2}+1\right)}\left(\frac{4 Q^{2}}{\lambda^{2}}\right)^{-\frac{\left((n-3) c^{2}+1\right.}{(2 n-4) c^{2}}} \\
\mathcal{Q}_{3} & =Q-\frac{c^{2} Q(n-2) \Gamma\left[\frac{(3 n-7) c^{2}+1}{(2 n-4) c^{2}}\right] \Gamma\left[\frac{1-c^{2}}{(2 n-4) c^{2}}\right]}{\left((n-3) c^{2}+1\right) \Gamma\left[\frac{(n-3) c^{2}+1}{(2 n-4) c^{2}}\right] \Gamma\left[\frac{(2 n-5) c^{2}+1}{(2 n-4) c^{2}}\right]} \\
\mathcal{H}_{3} & =\frac{\lambda^{2}}{4 Q c^{2}}+\frac{\left(c^{2}-1\right)}{\left((2 n-3) c^{2}-1\right)}\left(\frac{4 Q^{2}}{\xi^{2 n-4} \lambda^{2}}\right)^{-1}\left(Q-\mathcal{Q}_{3}\right) .
\end{aligned}
$$

Again all the solutions are singular at $r=0$. The Fig 3 says the detailed asymptotic structure of the spacetime. The general form of the asymptotic metric would be

$$
d s^{2}=r^{\frac{2 c^{2}}{1+c^{2}}}\left(-L_{0} r^{\frac{2-2 c^{2}}{1+c^{2}}} d t^{2}+d \Omega_{k}^{2}\right)+\frac{1}{L_{0} r^{\frac{2}{1+c^{2}}}} d r^{2}
$$




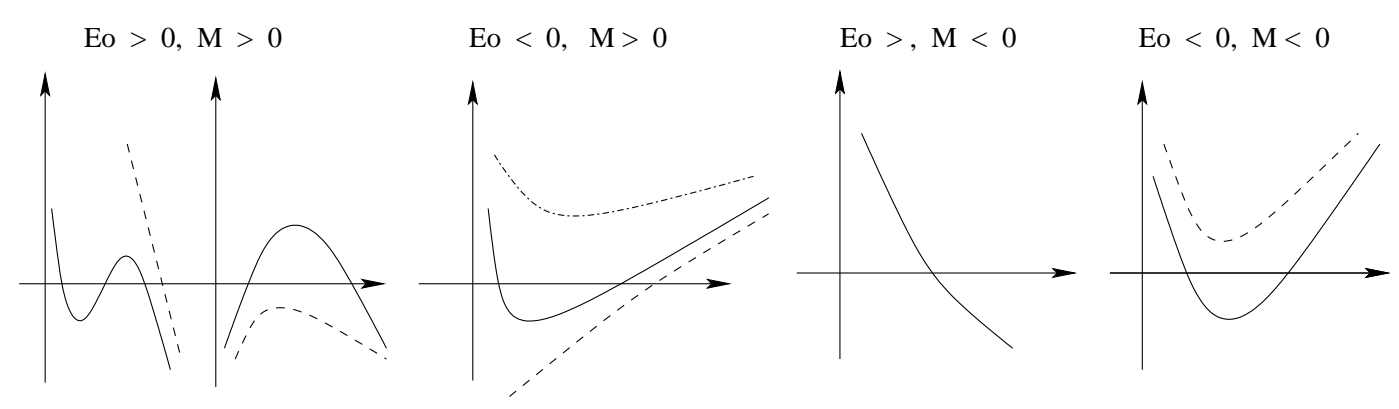

Figure 4: $F(R)$ for type-I solution.

So, it is to be noted that $L_{0}$ characterise the nature of asymptotic metric for full range of $c^{2}$.

The structure of the metric near $r=0$ is determined by the value of $c^{2}$. When $c^{2}>1$ it is the parameter $\mathcal{M}_{3}$ which plays the role. Where as if $c^{2}<1$ then singularity structure is determined by $\mathcal{Q}_{3}$. In this case also, the behaviour of $\mathcal{M}_{3}$ and $\mathcal{Q}_{3}$ is same as that of Type-II solution.

It is clear from the plots, when $L_{0}\langle 0, M>0$, we can have either Schwarzschild or $\mathrm{RN}$ type black hole in bulk spacetime depending upon the value of various parameters. Otherwise, it has naked singularity.

\section{Domain wall dynamics}

Dynamics of the domain wall is governed by the Hubble kind of expansion equation with respect to the domain wall observe. So, in general the equation looks like

$$
\dot{R}^{2}+F(R)=0
$$

where, '!' is derivative with respect to the domain wall proper time $\tau$. The expression for $F(R)$ is

$$
F(R)=N(R) R^{\prime 2}-\frac{\bar{V}_{0}^{2}}{4(n-2)^{2}} R^{2},
$$

where "prime" is derivative with respect to $\mathrm{r}$. So, the equation is like a particle moving in potential $F(R)$. So, in what follows we will be studying different types of potential encountered by the domain wall during the course of its motion.

Type-I potential: The expression for the potential is given below

$$
F(R)=N(R)-\frac{\bar{V}_{0}^{2}}{4(n-2)^{2}} R^{2} .
$$

The form of the potential as shown in Fig. 4 is like metric function $N(R)$ with an asymptotic modification by the domain wall tension.

Now, we will try to analyze the motion of the domain wall in various limits of the scale factor or distance of the wall along the bulk radial co-ordinate. Rather than writing down the exact expression for the potential, it is better to see the limiting cases. So, in the large $R$ limit, the equation of motion would be

$$
H^{2}=\frac{\dot{R}^{2}}{R^{2}}=-\frac{k}{R^{2}}+E_{0}+2 M R^{-(n-1)}-\mathcal{O}\left(R^{-(2 n-4)}\right),
$$


where

$$
E_{0}=\frac{2 L_{0}}{(n-1)(n-2)}+\frac{\bar{V}_{0}^{2}}{4(n-2)^{2}}
$$

For small $R$ limit,

$$
\begin{aligned}
H^{2}=-\frac{k}{R^{2}}+2 \mathcal{M} R^{-(n-1)} & +\frac{16 \lambda \mathcal{Q}}{(n-1)(n-2)} R^{-(n-2)} \\
& +\frac{2 \mathcal{V}}{(n-1)(n-2)}+\frac{\bar{V}_{0}^{2}}{4(n-2)^{2}}+\mathcal{O}\left(R^{n-2}\right) .
\end{aligned}
$$

So, from the above equation it is clear that for flat spatial section $k=0$, with $\left(\mathcal{M}_{1}>\right.$ 0 ), the domain wall is radiation dominated in the early epoch followed by the matter domination and cosmological constant domination respectively. Where as the bad feature is that during the course of its motion, domain wall hits the bulk singularity at $R=0$ for finite period of time. On the other hand, with the parameters being $\mathcal{M}_{1}<0$ but $M>0$, the domain wall starts from a finite value of the scale factor with matter energy domination followed by late time cosmological constant domination for $E_{0}>0$. Domain wall never reaches the bulk singularity in this case. A kind of bouncing universe scenario appears in th domain wall world volume (recent study [25]). For the first case, the bulk space time may either be non-static space time with naked singularity or a Schwarzschild black hole. For the second case, the bulk can have a de-Sitter horizon or it can be RN black hole depending upon the sign of $V_{0}$. Furthermore, a special range of value of parameters exist so that the domain wall depicts a periodic universe between the two horizons of the bulk black hole background. During this stage of evaluation, the domain wall has a finite period of inflation near the minimum of the potential followed by the standard deceleration. This case happens for both the cases $M>0, E_{0}>0$ and $M<0, E_{0}<0$ as is seen from the plot as well.

For every case, asymptotic dynamics of the domain wall is fixed by the interplay between the bulk cosmological constant and the brane tension. So, if $E_{0}>0$, then domain wall undergoes exponential inflation

$$
R(\tau) \propto e^{\sqrt{E_{0}} \tau}
$$

Another interesting case is $M<0, E_{0}>0$, which again leads to bouncing cosmology. So, domain wall starts collapsing from infinity to a finite value of $R=R_{0}$ say. At this value, domain wall gets repelled back to infinity by the timelike singularity in the bulk. The value of $R_{0}$ may be inside the de-Sitter horizon or the inner horizon of a RN type black hole of the background spacetime.

Type-II potential: The domain wall strictly restricted to be of spatially flat metric $(k=0)$. However, we note below the Hubble equation of the domain wall for in large $R$ limit,

$$
H^{2}=P_{0} R^{-2 c^{2}}+\mathcal{O}\left(R^{-\left(n-1+c^{2}\right)}\right)
$$

where

$$
P_{0}=\left[\frac{2 L_{0}}{n-1-c^{2}}+\frac{\bar{V}_{0}^{2} \Omega^{\prime}}{4(n-2)}\right]
$$




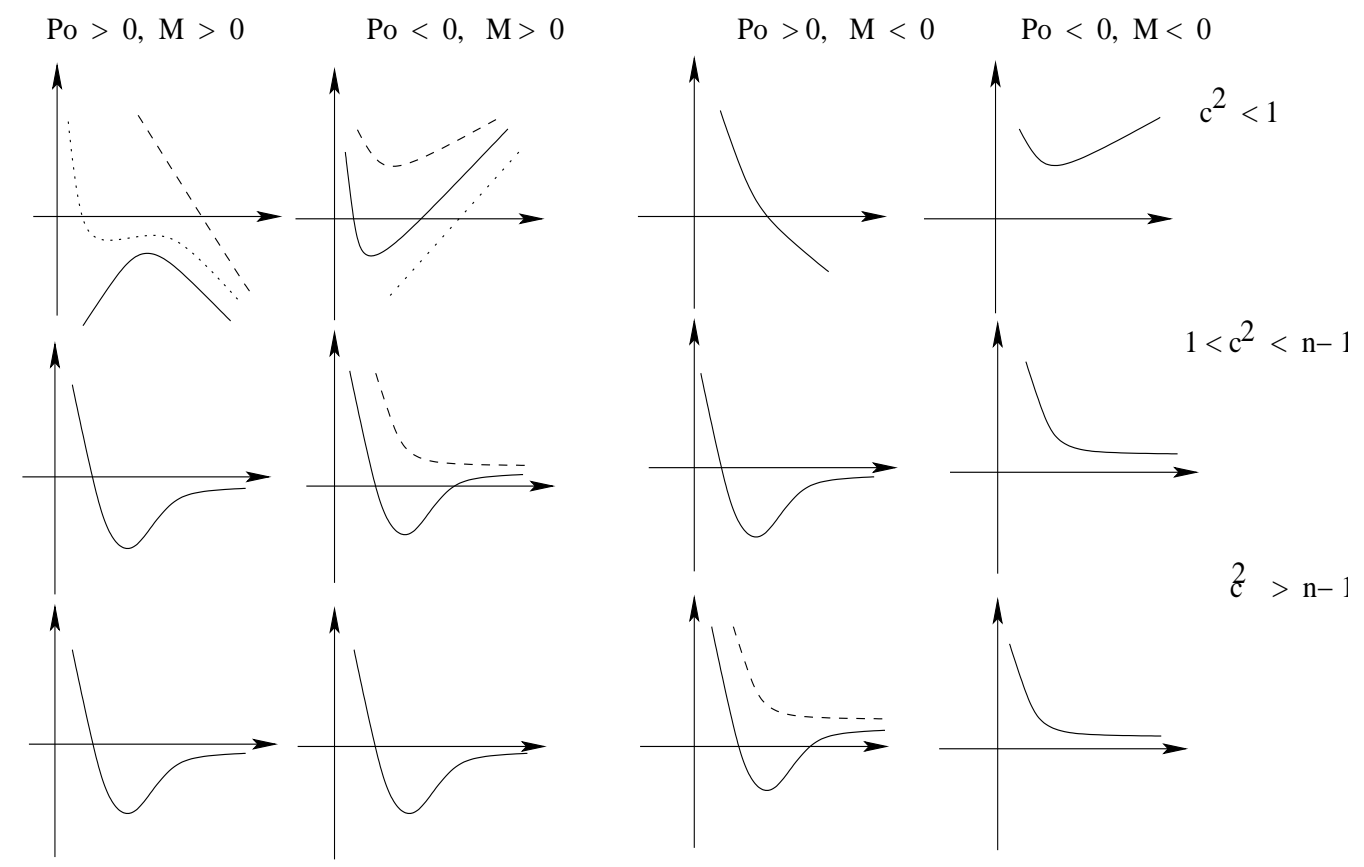

Figure 5: $\mathrm{F}(\mathrm{R})$ for type-II solution. For the range $n-1<c^{2}<n-3$, the qualitative form of the potential is same as for $1<c^{2}<n-3$

On the other hand for small $R$ limit, the expression for the above Hubble like equation turns out to be

$$
\begin{aligned}
H^{2}=2 \mathcal{M}_{2} R^{-\left(n-1+c^{2}\right)} & +\frac{16 \lambda \Omega^{\prime} \mathcal{Q}_{2}}{n-1-c^{2}} R^{-\left(n-2+2 c^{2}\right)}+\left(\frac{2 \mathcal{V}}{n-1-c^{2}}+\frac{\bar{V}_{0}^{2} \Omega^{\prime}}{4(n-2)}\right) R^{-2 c^{2}} \\
& +\frac{8 \lambda \Omega^{\prime} \mathcal{H}_{2}}{n-1-c^{2}} R^{-\left(n-2-2 c^{2}\right)}+\mathcal{O}\left(R^{3 n-6-2 c^{2}}\right) .
\end{aligned}
$$

In this case, we have different possibilities for the form of the potential function depending upon the value of the set of parameters as is also seen from Fig. 5

Case-i) $F(R)$ is positive every where. So, in this case there is no dynamics of the domain wall.

Case-ii) $\mathrm{F}(\mathrm{R})$ is negative for finite rang of $\mathrm{R}$. For $P_{0}<0, M>0$ with $c^{2}<n-1$ and $P_{0}>0, M>0$ with $c^{2}>n-1$ we have thi this kind of behaviour. However, in this case, the potential has minimum which corresponds to a short period of inflation followed by the decelerated expansion. The domain wall has a period oscillation between two extreme value of the scale factor.

Case-iii) $\mathrm{F}(\mathrm{R})$ is positive for small $R$ and then negative for large $R$. Now depending upon the value of $c$, the asymptotic structure of the potential again is of two different types. As we note asymptotically $F(R) \sim-P_{0} R^{2-2 c^{2}}$. So, it is clear that for $P_{0}>0$, when $c^{2}>1, F(R)$ grows negatively clear from the plot also. For $c^{2}<0, F(R)$ tends to zero value from negative side. So, generally, domain wall starts from infinity and gets repelled by the time like singularity in the bulk from a finite value of $R$. Asymptotically, the dynamics is

$$
R(\tau) \propto \tau^{\frac{1}{c^{2}}}
$$



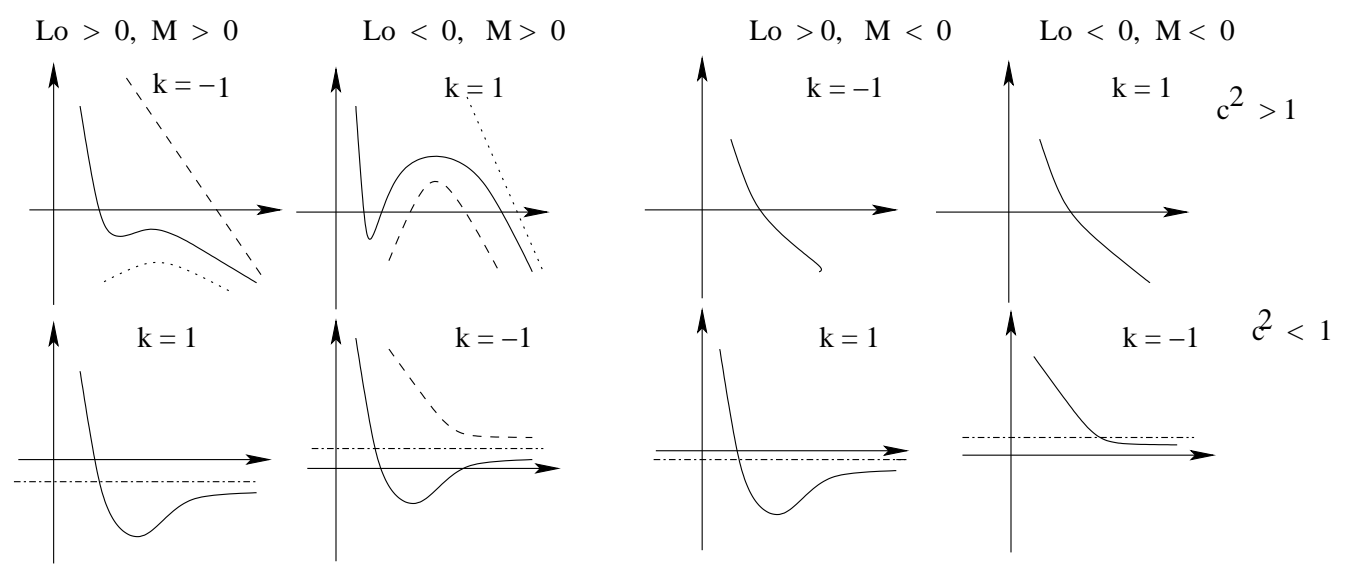

Figure 6: F(R) for type-III solution.

So, $c^{2}<1$ gives a late time accelerated expansion of the domain wall. For other case, expansion is decelerated. For all the cases we get bouncing universe scenarios.

Case-iv) $F(R)$ is negative every where. In this case, the domain wall has big-bang singularity at $R=0$. For $P_{0}>0, M>0$ and $c^{2}>1$, we have this form of the potential. Domain wall starts collapses from infinity all the way to bulk singularity.

Case-v) $F(R)$ is negative for small value of $R$ but positive for large value. This occurs only for $P_{0}<0, M>0$ irrespective of the value of $c^{2}$. In this potential domain wall emerges from a the white hole region of the bulk spacetime and gets halted at finite value of its scale factor and re-collapses into the black hole.

Type-III potential: The domain wall in this background strictly restricted to be of spatially non-flat metric $(k \neq 0)$. For large $R$ limit Hubble equation for the potential Eq 64 with this solution looks like

$$
H^{2}=\frac{\xi^{2} c^{4}}{R^{2}}\left[\frac{2 L_{0}}{(n-3) c^{2}+1}+2 M\left(\frac{R}{\xi}\right)^{-\frac{\left((n-3) c^{2}+1\right)}{c^{2}}}+\frac{\bar{V}_{0}^{2} \Omega^{\prime}\left(\frac{R}{\xi}\right)^{2-\frac{2}{c^{2}}}}{4(n-2)}\right]+\mathcal{O}\left(R^{-2 n-6}\right) .
$$

On the other hand for small $R$ limit, the expression for the above Hubble like equation turns out to be

$$
\begin{aligned}
H^{2} & =\frac{\xi^{2} c^{4}}{R^{2}}\left[2 \mathcal{M}_{3}\left(\frac{R}{\xi}\right)^{-\frac{(n-3) c^{2}+1}{c^{2}}}+\frac{16 \lambda \Omega^{\prime} \mathcal{Q}_{3}}{c^{2} \xi^{n-2}\left((n-1) c^{2}-1\right)}\left(\frac{R}{\xi}\right)^{-\frac{(n-4) c^{2}+2}{c^{2}}}+\frac{2 L_{0}}{(n-1) c^{2}+1}\right. \\
& \left.+\frac{\bar{V}_{0}^{2} \Omega^{\prime}\left(\frac{R}{\xi}\right)^{2-\frac{2}{c^{2}}}}{4(n-2) c^{2}}-\frac{8 \lambda^{2} \Omega^{\prime}\left(\frac{R}{\xi}\right)^{2-\frac{2}{c^{2}}}}{c^{2}\left((n-1) c^{2}-1\right)}-\frac{8 \lambda \Omega^{\prime} \mathcal{H}_{3}\left(\frac{R}{\xi}\right)^{n-\frac{2}{c^{2}}}}{c^{2}\left((n-1) c^{2}-1\right)}\right]+\mathcal{O}\left(R^{\frac{(3 n-6) c^{2}-2}{c^{2}}}\right) .
\end{aligned}
$$

As is seen from the plot Fig , qualitatively the form of potential can be categorized into two sets corresponding to the value of $c^{2}$. In one $c^{2}>1$, the potential tends to asymptotic negative infinity. This leads to asymptotically power law type inflation. In the small $R$ limit, domain wall can have bounce due to the presence of time like bulk singularity for full $\left(L_{0}, M\right)$ space or it can go all the way to bulk attractive spacelike singularity. 
In the other one $c^{2}<1$, the potential is asymptotically goes to some constant value depending on the value of $L_{0}$. The constant $L_{0}$ is shown in the plots by dot-dashed horizontal lines. For this, we have three kind of potential structures. In many respects, these are of the same form compare to type-II potential. But main difference is the value of the parameter $c^{2}$. It acts as a inverse of that of type-II case.

Asymptotically, the domain wall undergoes

$$
\begin{aligned}
& R(\tau) \propto \tau \quad \text { for } c^{2}<1 \text { and } L_{0}>0 \\
& R(\tau) \propto \tau^{c^{2}} \text { for } c^{2}>1 .
\end{aligned}
$$

So, for the first case, it is linear expansion with spherical spatial section. Where as for the second case, domain wall has power law inflation. For almost every case, domain wall has a bounce for a finite value of scale factor.

\section{Conclusion}

To summarize, in this report we have tried to study the Einstein-Born-Infeld-dilaton theory in presence of dynamic domain walls. We have first tried to find out the possible background solutions taking into account the domain wall back-reaction. We have analytically found three different types of solutions. The analytical study of these various metrics is very difficult. So, we have adopted the same line as in [22, 20] by plotting all the metric functions and studied its structure in various limits along the radial coordinate.

As was mentioned earlier, BI electromagnetic field has a critical value which is responsible for smoothing of pointlike singularity of the vector field. So, it is expected to have same kind of affects on the gravitational background in connection with the singularity at the origin. We have seen in our previous work [20] for Maxwell electromagnetic case which is basically $\lambda \rightarrow \infty$ limit of the present analysis that for the first three types of solutions the singularities were governed by the electric charge $\mathrm{Q}$ as expected. The singularities were in general timelike in those cases. On the other hand the present analysis shows that for finite value of $\lambda$, singularities are somewhat smoothed. As one can see explicitly for the first three solutions being one to one correspondence with $\lambda \rightarrow \infty$ case, singularities at $r=0$ are governed either by parameter $\mathcal{M}$ which is related to mass parameter $M$ or charge Q. Importantly, depending upon the various parameters, in these cases singularity can either be timelike or spacelike as also clear from various plots. However, in order to be more explicit, we demonstrated in the Table I that the nature of singularities appear in the different background metric solutions for different limits of $\lambda$.

\begin{tabular}{|c|c|c|}
\hline Type of Sol ${ }^{n}$ & $\begin{array}{l}\text { Maxwell EM field } \\
\qquad \lambda \rightarrow \infty\end{array}$ & $\begin{array}{l}\text { BI EM field } \\
\lambda \text { is finite }\end{array}$ \\
\hline Type-I & $Q^{2} r^{-(2 n-6)}$ & $\mathcal{M}_{1} r^{-(n-3)}$ \\
\hline Type-II & $Q^{2} r^{-\frac{2 n-6}{1+c^{2}}}$ & $\begin{array}{cc}\mathcal{M}_{2} r^{-\frac{n-3-c^{2}}{1+c^{2}}} & \text { for } c^{2}<1 \\
Q r^{-\frac{n-4}{1+c^{2}}} & \text { for } c^{2}>1\end{array}$ \\
\hline Type-III & $Q^{2} r^{-\frac{(2 n-6) c^{2}}{1+c^{2}}}$ & $\begin{array}{cc}\mathcal{M}_{3} r^{-\frac{(n-3) c^{2}-1}{1+c^{2}}} & \text { for } c^{2}>1 \\
Q r^{-\frac{(n-4) c^{2}}{1+c^{2}}} & \text { for } c^{2}<1\end{array}$ \\
\hline
\end{tabular}

Table 1: Comparison of various singularities up to sign 
It may also be noted that for any value of $\lambda$ the asymptotic structure i.e. in $r \rightarrow \infty$ limit, the background spacetime does not depend on it at all.

Finally, after getting details of the background spacetime we have tried to study the dynamics of the domain walls in those bulk spacetime configurations. In this case also, there exists specific relations among the various coupling parameters so that one can have static bulk spacetime background in consistent with the dynamic domain wall. As a direct consequence of drastic changes of the singularity behaviour in our present analysis compared to $\lambda \rightarrow \infty$ case [20], the dynamics of the domain wall near the singularity has also modified significantly follows from various plots.

In many cases again, we also have found to exist inflation for finite period of proper time with respect to domain wall world volume followed by standard decelerated expansion phase. Another important feature is the presence of negative energy density in domain wall scenario. In fact, the very presence of this negative energy density, plays the role of bounce for the domain wall avoiding bulk singularity. This has recently been discussed [25] for the first solution. But we have several solutions with different asymptotic behaviour as well as near singularity structure for the same kind of background field configurations. So, in that respect it might be interesting to study this bounce in details for the other non-trivial background solutions.

In the context of dark energy and dark matter in our universe, these various kind of induced unseen energy density on the wall may be interesting points to study. For example, this non-standard behavior may help us to construct dark matter and dark energy [26] model building [27] in solving discrepancies with standard general relativity predictions for the galaxy rotation curves [28, late time acceleration of the universe [29], gravitational lensing [30]. As an another possible interesting extension of this work would be to analyze stability under perturbation in the domain wall world volume. An interesting point to analyze would be whether all these types of solutions are compatible in addition to external matter sources such as radiation and baryonic matter, restricted to the domain world volume.

\section{Acknowledgment}

The referee's valuable comments and suggestions are gratefully acknowledged.

\section{References}

[1] D. W. Joseph, Phys. Rev. 126, 319 (1962).

[2] K. Akama, Lect. Notes Phys.176, 267 (1982) hep-th/0001113; K. Akama, Prog. Theor. Phys. 60, 1900 (1978); K. Akama, Prog. Theor. Phys. 78, 184 (1987); 79, 1299 (1988); 80, 935 (1988); K. Akama and T. Hattori, Mod. Phys. Lett. A15, 2017 (2000).

[3] V.A. Rubakov and M.E. Shaposhnikov, Phys. Lett. B125, 136 (1983); M. Visser, Phys. Lett. 159B, 22 (1985).

[4] P. Laguna-Castillo and R. A. Matzner, Nucl. Phys. B282, 542 (1987); E. J. Squires, Phys. Lett. B167, 286 (1986); G. W. Gibbons and D. L. Wiltshire, Nucl. Phys. B287, 717 (1987); J.M. Overduin and P.S. Wesson, Phys. Rept. 283, 303 (1997). 
[5] L. Randall, R. Sundrum, Phys. Rev. Lett. 83, 3370 (1999) hep-ph/9905221; Phys. Rev. Lett. 83, 4690 (1999) hep-th/9906064.

[6] G. Dvali, M. Shifman, Phys. Lett. B396, 64 (1997) hep-th/9612128]; Nucl. Phys. B504, 127 (1996) hep-th/9611213].

[7] M. Cvetic and H. H. Soleng, Phys. Rept. 282, 159 (1997) hep-th/9604090|.

[8] J. Hughes, J. Liu and J. Polchinski, Phys. Lett. B180, 370 (1986).

[9] A. Burt A. Ovrut and D. Waldram, Phys. Rev. D61, 023506 (2000) hep-th/9902071, ibid Phys. Rev. D60, 086001 (1999) hep-th/9806022.

[10] J. Polchinski, Phys. Rev. Lett. 75, 4724 (1995) hep-th/9510017.

[11] P. Kraus, JHEP 9912, 011 (1999) hep-th/9910149].

[12] T. Nihei, Phys. Lett. B465,81 (1999), hep-ph/9905487]; C. Csaki, M. Graesser, C. Kolda and J. Terning, Phys. Lett. B426,34 (1999), hep-ph/9906513; P. Binetruy, C. Deffayet, U. Ellwanger and D. Langlois, Phys. Lett. B477,285 (2000), hep-th/9910219]; D. Ida, JHEP 0009,014 (2000), [gr-qc/9912002]; C. Bercelo and M. Visser, Phys. Lett. B482,183 (2000), [hep-th/0004056]; L. Anchordoqui, C. Nunez and K. Olsen, JHEP 0010,050 (2000), [hep-th/0007064]; P. Bowcock, C. Charmousis and R. Gregory, Class. Quant. Grav. 17,4745 (2000), [hep-th/0007177]; C. Csaki, J. Erlich and C. Grojean, Nucl. Phys. B604,312 (2001), hep-th/0012143]; Y. S. Myung, hep-th/0103241. D. H. Coule, Class. Quant. Grav. 18 (2001) 4265; J. P. Gregory and A. Padilla, Class. Quant. Grav. 19,4071 (2002), hep-th/0204218. S. Nojiri, S. D. Odintsov and S. Ogushi, hep-th/0205187.

[13] W. Israel, Nuovo Cimento, B44, 1 (1966), Erratum: B48, 463 (1967).

[14] S. Mukherji and M. Peloso, Phys. Lett. B547, 297 (2002) hep-th/0205180. A. Biswas, S. Mukherji and S. Sekhar Pal, Int. J. Mod. Phys. A19, 557 (2004) hep-th/0301144; A. Biswas and S. Mukherji, JCAP 0602, 002 (2006) [hep-th/0507270];S. Mukherji, S. Pal, arXiv:0806.2507 [gr-qc].

[15] M. Novello and S.E.Perez Bergliaffa, arXiv:0802.1634 [astro-ph] and references there in; J. L. Hovdebo and R. C. Myers, JCAP 0311, 012 (2003) [hep-th/0308088]. C.P. Burgess, F. Quevedo, R. Raba, G. Tasinato and I. Zavala, JCAP, 0402, 008 (2004) hep-th/0310122 ; M. R. Setare, F. Darabi, Int. J. Mod. Phys. D16, 1563 (2007) hep-th/0605081. G. De. Risi, Phys. Rev. D7, 044030 (2008).

[16] E. S. Fradkin and A.A. Tseytlin, Phys. Lett. B160, 69 (1985).

[17] R. G. Leigh, Mod. Phys. Lett. A4, 2767 (1989).

[18] T. Kr. Dey, Phys. Lett. B595, 484 (2004)|hep-th/0406169|; R. Cai, D. Pang and A. Wang, Phys. Rev. D70, 124034 (2004) hep-th/0410158]. 
[19] D. L. Wiltshire, Phys. Rev. D38, 2445 (1988); D. A. Rasheed, arXiv:hep-th/9702087; M. Cataldo and A. Garcia, Phys. Lett. B456, 28 (1999)[hep-th/9903257]; S. Fernando and D. Krug, Gen. Rel. Grav. 35, 129 (2003)[hep-th/0306120]; T. Tamaki, JCAP 0405, 004 (2004) [gr-qc/0310099]; S. Fernando, Gen. Rel. Grav. 37, 585 (2005) hep-th/0407062]; S. Fernando, C. Holbrook, Int. J. Theor. Phys. 45, 1630 (2006) |hep-th/0501138|; E. F. Eiroa, Phys. Rev. D73 043002 (2006) [gr-qc/0511065]; S. Fernando, Phys. Rev. D74, 104032 (2006) [hep-th/0608040]; A. Sheykhi, N. Riazi, Phys. Rev. D75, 024021 (2007) [hep-th/0610085]; M. H. Dehghani, S. H. Hendi, A. Sheykhi and H. R. Sedehi, JCAP 0702, 020 (2007) hep-th/0611288]; X. Gao, JHEP 0711, 006 (2007) arXiv:0708.1226] [hepth]; S. Yun, arXiv:0706.2046 [hep-th]; B. Chandrasekhar, H. Yavartanoo and S. Yun, Phys. Lett. B660, 392 (2008)[hep-th/0611240]; A. Sheykhi, Phys. Lett. B662, 7 (2008) arXiv:0710.3827] [hep-th]; A. Sheykhi, arXiv:0801.4112 [hep-th]; O. Miskovic and R. Olea arXiv:0802.2081 [hep-th]; Y. S. Myung, Y. Kim and Y. Park, arXiv:0804.0301 [gr-qc]; Y. S. Myung, Y. Kim and Y. Park, arXiv:0805.0187 [gr-qc]; S. S. Yazadjiev, Phys. Rev. D72, 044006 (2005) [hep-th/0504152]; M.H. Dehghani, N. Bostani and S.H. Hendi, arXiv:0806.1429 [gr-qc].

[20] D. Maity, arXiv:0806.2041[hep-th].

[21] T. Tamaki and T. Torii, Phys. Rev. D62, 061501 (2000) gr-qc/0004071]; Clement and D. Galtsov, Phys. Rev. D62, 124013 (2000) hep-th/0007228].

[22] H.A. Chamblin and H.S. Reall, Nucl. Phys. B 562, 133 (1999); A. Chamblin, M. J. Perry and H. S. Reall, JHEP 9909, 014 (1999) hep-th/9908047.

[23] M.H. Dehghani et al Phys. Rev.D77, 104025 (2008) arXiv:0802.2637] [hep-th]; Z. Guo, N. Ohtaa and T. Toriib, arXiv:0806.2481[gr-qc].

[24] L. F. Abbot and S. Deser, Nucl. Phys. B195, 76 (1982); S. W. Hawking and G. T. Horowitz, Class. Quant. Grav. 13, 1487 (1996).

[25] S Mukherji and S. Pal, arXiv:0806.2507 [gr-qc].

[26] L. Bergstrom, Rept. Prog. Phys. 63, 793 (2000); F. Combes, New Astron. Rev. 46, 755 (2002).

[27] S. Pal, S. Bharadwaj and S. Kar, Phys. Lett. B609, 194 (2005) gr-qc/0409023]; C. G. Boehmer and T. Harko, Class. Quant. Grav. 24, 3191 (2007) [0705.2496 [gr-qc]].

[28] J. J. Binney and S. Tremaine, Galactic Dynamics, Princeton University Press, Princeton (1987); M. Persic, P. Salucci and F. Stel, Mon. Not. Roy. Astron. Soc. 281, 27 (1996); A. Berriello and P. Salucci, Mon. Not. Roy. Astron. Soc. 323, 285 (2001); Y. Safue and V. Rubin, Ann. Rev. Astron. Astrophys. 39, 137 (2001).

[29] A. G. Riess et al., Astron. J. 116, 1009 (1998); S. Perlmutter et al., Bull. Am. Astron. Soc. 29, 1351 (1997); S. Perlmutter et al., Astrophys. J. 517, 565 (1997); J. L. Tonry et al., Astrophys. J. 594, 1 (2003); S. Bridle, O. Lahav, J. P. Ostriker and P. J. Steinhardt, Science, 299, 1532 (2003); C. Bennet et al., Astrophys. J. Suppl. Ser. 148, 1 (2003) astro-ph/0302207]; G. Hinshaw et al., Astrophys. J. Suppl. Ser. 148, 
135 (2003) astro-ph/0302217; A. Kogut et al., Astrophys. J. Suppl. Ser. 148, 161 (2003) astro-ph/0302213|; D. N. Spergel et al., Astrophys. J. Suppl. Ser. 148, 175 (2003) astro-ph/0302209].

[30] P. Schneider, J. Ehlers and E. Falco, Gravitational lenses , Springer Verlag, Berlin (1992) 